\title{
Three Dimensional Digital Simulation and Autonomous Walking Control for Eight-Axis Biped Robot
}

\author{
Yasutaka Fujimoto Atsuo Kawamura \\ Department of Electrical and Computer Engineering, Yokohama National University \\ 156 Tokiwadai, Hodogaya-ku, Yokohama 240 JAPAN
}

\begin{abstract}
First, the three dimensional precise simulation method for biped robot has been proposed, which is the extension of the open link manipulator simulation method and the contact simulation method of rigid body mechanics. The proposed simulation model can be said to be mathematically exact, thus this approach enables the essential investigation for control algorithms of biped locomotion. This approach has advantages in the less cost compared with the experimental demonstration.

Second, the autonomous walking control with hierarchical structure has been proposed in which three dimensional inverted pendulum model is considered as the motion of gravity center, and the tip position of non-support leg are controlled autonomously. Both of references can be simultaneously transformed to the joint space reference, then they are accurately realized by the robust-servo controller.

Third, the proposed walking control is investigated by the proposed simulation method, and the stable walking motion is confirmed.
\end{abstract}

\section{Introduction}

The biped locomotion is necessary for future human type robots when robots work in the human-life space. Besides the practical aspects, the nonlinearlity and unstability are also interesting from the viewpoint of control theory.

A large number of control schemes of biped walking have been proposed and have been experimentally demonstrated $^{[8][9]}$. There are, however, unknown disturbances in the experiments, which makes it difficult to inspect these algorithm's effectiveness. Such a complex control algorithm should be firstly inspected by a precise simulation with an ideal environment. After that, the control method should be extended to real robots. This approach takes the less cost than experimental trial-and-error and it enables a control algorithm to be furtherly refined.
In some previous works, simple simulation models have been considered ${ }^{[1][2]}$. They are, however, planar link models and the constraints to the ground are under the assumptions of no slipping and no impact reaction. These simplifications cause substantial loss of stability phenomena of the practical biped locomotion, which results in a wide gap between simulation results and experimental results.

On the other hand, very complex simulation method including joint compliance have proposed ${ }^{[3]}$. That model, however, seems too complex to investigate the fundamental of walking control.

In this paper, we propose a novel precise digital simulation method based on a combination of a general manipulator simulation ${ }^{[12]}$ and a contact simulation of rigid body mechanics ${ }^{[14]}$. In this method, 3-dimensional contact to the ground is expressed as impact reaction, the constrained motion of which becomes more real than the simple model and more ideal than the complex model. Using this precise simulation model, we have proposed a new walking control algorithm including on-line type autonomous motion design and robust-servo based motion control.

\section{Simulation Scheme}

\subsection{Dynamics of Free-falling Manipula- tor}

A biped robot is considered as a free-fall manipulator which does not have fixed-point but has interaction to the ground. A dynamics simulation scheme of a free-fall manipulator can be easily obtained from an expansion of general manipulator simulation scheme ${ }^{[12]}$.

In order to express states of posture (3DOF of position and 3DOF of rotation), virtual links with no-mass and no-length are added on the base link of manipulator as shown in Fig.1. Using the joint angles (3DOF) and the base position (3DOF) of the virtual links, we can deal with total 6DOF states of the posture of the robot. This expression of $6 \mathrm{DOF}$ states is simpler than 
the another approach, for example, using position of center of gravity.

The global motion dynamics can be expressed as follows,

$$
\begin{aligned}
\boldsymbol{H}(\boldsymbol{x}) \ddot{\boldsymbol{x}}+\boldsymbol{b} & =\boldsymbol{u}+\boldsymbol{K}(\boldsymbol{x}) \boldsymbol{f}_{E X T} \\
\boldsymbol{b} & =\boldsymbol{C}(\boldsymbol{x}, \dot{\boldsymbol{x}}) \dot{\boldsymbol{x}}+\boldsymbol{G}(\boldsymbol{x})
\end{aligned}
$$

Here, $\boldsymbol{x}^{T}=\left[\boldsymbol{p}_{0}^{T}, \boldsymbol{q}^{T}\right], \boldsymbol{u}^{T}=\left[\boldsymbol{f}_{0}^{T}, \boldsymbol{\tau}^{T}\right]$, and another parameters are,

$$
\begin{aligned}
& p_{0} \quad: \quad 3 \times 1 \text { vector specifying position of } \\
& \text { virtual base link } \\
& \boldsymbol{q} \quad: \quad(N+3) \times 1 \text { vector specifying angular } \\
& \text { of joints including virtual ones } \\
& \boldsymbol{H}(\boldsymbol{x}):(N+6) \times(N+6) \text { inertia matrix } \\
& f_{0}: 3 \times 1 \text { force vector generated in virtual } \\
& \text { base link } \\
& \tau \quad:(N+3) \times 1 \text { torque vector generated by } \\
& \text { actuator } \\
& \boldsymbol{C}(\boldsymbol{x}, \dot{\boldsymbol{x}}) \quad: \quad(N+6) \times(N+6) \text { matrix specifying } \\
& \text { centrifugal and Coriolis effects } \\
& \boldsymbol{G}(\boldsymbol{x}) \quad: \quad(N+6) \times 1 \text { vector specifying gravity } \\
& \text { effect } \\
& \boldsymbol{K}(\boldsymbol{x}) \quad: \quad(N+6) \times 2 M \text { transformer matrix from } \\
& \text { external forces to state forces } \\
& f_{E X T}: 2 M \times 1 \text { vector of external forces; } \\
& \text { a contact point produces two kind of } \\
& \text { forces, vertical and horizontal to the } \\
& \text { ground. } \\
& N \quad \text { : number of real joints } \\
& M \quad \text { : number of contact point; } \\
& \text { time-dependent }
\end{aligned}
$$

In order to simulate above dynamics, we integrate (1) about $\ddot{\boldsymbol{x}}$ numerically, after solving (1) for the accelerations given $\boldsymbol{x}, \dot{\boldsymbol{x}}$, and the input $\boldsymbol{u}^{T}=\left[\begin{array}{ll}0^{T} & \boldsymbol{\tau}^{T}\end{array}\right]$. $\boldsymbol{H}(\boldsymbol{x})$ and $\boldsymbol{b}$ can be obtained by inverse dynamics calculation using Newton-Euler formulation ${ }^{[13]}$, that is, given $\boldsymbol{x}, \dot{\boldsymbol{x}}$ and $\ddot{\boldsymbol{x}}$ solve for $\boldsymbol{u}$.

In fact, $\boldsymbol{H}(\boldsymbol{x})$ can be calculated by solving inverse dynamics with setting $\boldsymbol{x}$ to the current state, $\ddot{\boldsymbol{x}}=\boldsymbol{e}_{j}$, and ignoring centrifugal and Coriolis forces, gravity effects, external forces ${ }^{[12]}$. Here, $\boldsymbol{e}_{j}$ means an unit vector with its $j$ th element equal to 1 and others are 0 . The solution about $\boldsymbol{u}$ corresponds to the $j$ th column of $\boldsymbol{H}$. The biasing vector $\boldsymbol{b}$ can be also computed by setting $\ddot{x}=0$.

The inverse dynamics can be calculated by following recursive equations ${ }^{[13]}$. The angular velocity and acceleration of virtual base link $\omega_{0}, \dot{\omega}_{0}$ are equal to 0 because they are constantly parallel with the ground, then the angular velocity $\boldsymbol{\omega}_{i}$, the angular acceleration $\dot{\boldsymbol{\omega}}_{i}$, the acceleration of the origin $\ddot{\boldsymbol{p}}_{i}$, the acceleration of

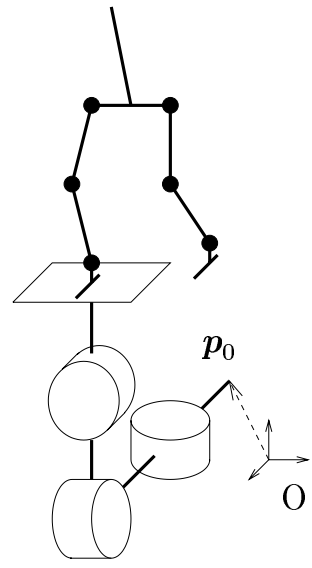

Figure 1: Virtual links.

the center of mass $\ddot{\boldsymbol{r}}_{i}$, the total force $\boldsymbol{F}_{i}$, and the total moment $\boldsymbol{N}_{i}$ of the $i$ th link can be recurrently obtained as follows, when $\ddot{p}_{0}, \ddot{q}_{i}, \dot{q}_{i}$ and $q_{i}$ are given.

$$
\begin{aligned}
\boldsymbol{\omega}_{0} & =\dot{\boldsymbol{\omega}}_{0}=0 \\
\boldsymbol{\omega}_{i} & =\boldsymbol{\omega}_{i-1}+\boldsymbol{z}_{i-1} \dot{q}_{i} \\
\dot{\boldsymbol{\omega}}_{i} & =\dot{\boldsymbol{\omega}}_{i-1}+\boldsymbol{z}_{i-1} \ddot{q}_{i}+\boldsymbol{\omega}_{i-1} \times \boldsymbol{z}_{i-1} \dot{q}_{i} \\
\ddot{\boldsymbol{p}}_{i} & =\dot{\boldsymbol{\omega}}_{i} \times \boldsymbol{p}_{i}^{*}+\boldsymbol{\omega}_{i} \times\left(\boldsymbol{\omega}_{i} \times \boldsymbol{p}_{i}^{*}\right)+\ddot{\boldsymbol{p}}_{i-1} \\
\ddot{\boldsymbol{r}}_{i} & =\dot{\boldsymbol{\omega}}_{i} \times \boldsymbol{s}_{i}+\boldsymbol{\omega}_{i} \times\left(\boldsymbol{\omega}_{i} \times \boldsymbol{s}_{i}\right)+\ddot{\boldsymbol{p}}_{i} \\
\boldsymbol{F}_{i} & =m_{i} \ddot{\boldsymbol{r}}_{i} \\
\boldsymbol{N}_{i} & =\boldsymbol{J}_{i} \dot{\boldsymbol{\omega}}+\boldsymbol{\omega} \times\left(\boldsymbol{J}_{i} \boldsymbol{\omega}_{i}\right)
\end{aligned}
$$

Here, $\boldsymbol{p}_{i}^{*}$ denotes $\boldsymbol{p}_{i}-\boldsymbol{p}_{i-1}, \boldsymbol{z}_{i}$ denotes the direction of the joint $i+1$, and $s_{i}$ denotes the center of mass with respect to the origin of link $i$ coordinates. The gravity effect can be considered by adding a gravity acceleration on $\ddot{p}_{0}$, the acceleration of the virtual base link.

Then, $\boldsymbol{f}_{i}$ and $\boldsymbol{n}_{i}$, the force and moment exerted on link $i$ by link $i-1$, and the torque on $i$ th joint $\tau_{i}$ can be calculated as follows,

$$
\begin{aligned}
\boldsymbol{f}_{i}= & \boldsymbol{F}_{i}+\boldsymbol{f}_{i+1}+\sum_{j \in M_{i}} \boldsymbol{u}_{j} f_{E X T j} \\
\boldsymbol{n}_{i}= & \boldsymbol{N}_{i}+\boldsymbol{n}_{i+1}+\left(\boldsymbol{p}_{i-1}-\boldsymbol{r}_{i}\right) \times \boldsymbol{f}_{i} \\
& +\left(\boldsymbol{p}_{i}-\boldsymbol{r}_{i}\right) \times \boldsymbol{f}_{i+1} \\
\tau_{i}= & \boldsymbol{z}_{i-1} \cdot \boldsymbol{n}_{i}-D_{i} \dot{q}_{i}
\end{aligned}
$$

Here, $f_{E X T} j$ is the $j$ th external force, $\boldsymbol{u}_{j}$ is its direction as shown in Fig.2, $M_{i}$ is a set of index number of external forces which are imposed on link $i$, and $D_{i}$ is the viscous damping coefficient of joint $i$. As a result, we can obtain the virtual force $f_{0}$ and the joint torque $\boldsymbol{\tau}$ given $\boldsymbol{x}, \dot{\boldsymbol{x}}$, and $\ddot{\boldsymbol{x}}$. Above equations can be efficiently calculated by denoting the parameter of link $i$ with respect to link $i+1$ coordinates $^{[13]}$. 


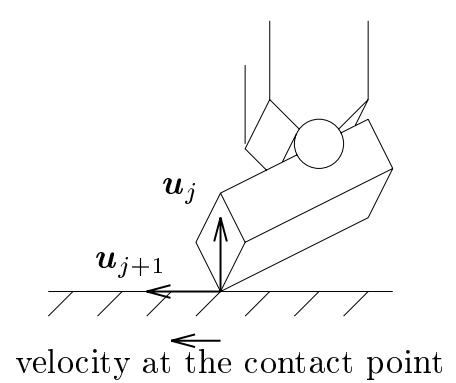

Figure 2: Directions of external forces.

\subsection{Calculation of Constrained Force}

In order to support the biped robot on the ground, the appropriate reaction forces from the ground should be determined at each time step in the simulation. In this paper, only impact forces between the foot and the ground are considered as the interaction forces. In general, springs and dampers model at the contact points are introduced as such a collision interaction. That simulation model, however, requires more shorter time step than the mechanical system itself does. Therefore we introduce a plastic collision model ${ }^{[14]}$, which is the kinetic energy minimization satisfying some constrains and inequalities. In the motion equation (1), the external forces $\boldsymbol{f}_{E X T}$ play the role of Lagrange multipliers. Thus, the main purpose is to solve $\boldsymbol{f}_{E X T}$ fulfilling appropriate conditions. In addition, a motion with the continuous plastic collision behaves an exact ideal constrained motion, then the biped robot can be supported by considering only plastic collision forces as the reaction forces.

When the external impulsive force $\Delta f$ are imposed in the system (1), the following equation is realized.

$$
\boldsymbol{H}(\boldsymbol{x})\left(\dot{\boldsymbol{x}}_{+}-\dot{\boldsymbol{x}}_{-}\right)=\boldsymbol{K}(\boldsymbol{x}) \Delta \boldsymbol{f}+\Delta \boldsymbol{f}_{b}
$$

Here, $\dot{\boldsymbol{x}}_{+}$and $\dot{\boldsymbol{x}}_{-}$denote the velocity after the collision and before the collision, respectively. $\Delta f_{b}$ denotes the uncontrollable impulse force, that is, $\Delta f_{b} \approx$ $\left(\boldsymbol{u}-\boldsymbol{b}\left(\boldsymbol{x}, \dot{\boldsymbol{x}}_{-}\right)\right) \Delta t$. Thus, the kinetic energy after the collision can be calculated as follows,

$$
\begin{gathered}
\frac{1}{2} \dot{\boldsymbol{x}}_{+}^{T} \boldsymbol{H} \dot{\boldsymbol{x}}_{+}=\frac{1}{2} \dot{\boldsymbol{x}}_{-}^{T} \boldsymbol{H} \dot{\boldsymbol{x}}_{-}+\frac{1}{2} \Delta \boldsymbol{f}^{T} \boldsymbol{K}^{T} \boldsymbol{H}^{-1} \boldsymbol{K} \Delta \boldsymbol{f} \\
+\left(\dot{\boldsymbol{x}}_{-}+\boldsymbol{H}^{-1} \Delta \boldsymbol{f}_{b}\right)^{T} \boldsymbol{K} \Delta \boldsymbol{f}+\frac{1}{2} \Delta \boldsymbol{f}_{b}^{T} \boldsymbol{H}^{-1} \Delta \boldsymbol{f}_{b}
\end{gathered}
$$

The plastic collision is defined as the energy minimization with given conditions. Therefore, impact force $\Delta f$ can be obtained by solving the following quadratic programming (QP) problem.

$$
\text { minimize } \quad \frac{1}{2} \Delta \boldsymbol{f}^{T} \boldsymbol{K}^{T} \boldsymbol{H}^{-1} \boldsymbol{K} \Delta \boldsymbol{f}
$$

$$
\begin{array}{ll} 
& +\left(\dot{\boldsymbol{x}}_{-}+\boldsymbol{H}^{-1} \Delta \boldsymbol{f}_{b}\right)^{T} \boldsymbol{K} \Delta \boldsymbol{f} \\
\text { subject to } & \Delta f_{N i} \geq 0 \\
& -\mu \Delta f_{N i} \leq \Delta f_{F i} \leq \mu \Delta f_{N i}
\end{array}
$$

Here, $\Delta f_{N i}$ and $\Delta f_{F i}$ denote the vertical force and the friction force on the $i$ th contact point, respectively. In advance, $\boldsymbol{K}$ can be obtained by solving inverse dynamics with setting $\boldsymbol{x}$ and $\dot{\boldsymbol{x}}$ to its current state, $\boldsymbol{f}_{E X T}=$ $\boldsymbol{e}_{j}, \ddot{\boldsymbol{x}}=0$, and ignoring gravity effects and centrifugal and Coriolis effects. The direction of contact force $\boldsymbol{u}_{j}$ in (9) is determined to the vertical, if it is vertical force, or the projection of velocity of contact point onto the ground plane, if it is friction force. The assumption that the direction of friction force corresponds to the projection of velocity onto the ground is usually adequate if the time step is enough short. Thus, we can deal with a 3 -dimensional Coulomb friction problem (usually it becomes quadratic condition, $\Delta f_{F i x}^{2}+$ $\Delta f_{F i y}^{2} \leq \mu^{2} \Delta f_{N i}^{2}$ ) as linear condition (16), that is suitable for mathematical programming problems. The condition (15) indicates that the vertical force is physically nonnegative.

Using the complementary pivot methods ${ }^{[15]}$ to solve this QP problem each time step, we can simulate the biped robot motion (1) with its solution of constrained force $\Delta f$.

Characteristics of Solution The solution of QP problem (14) with conditions (15) and (16) satisfy the following necessary and sufficient conditions (KuhnTucker conditions).

$$
\begin{aligned}
\Delta f_{N i}^{*} & \geq 0 \\
\boldsymbol{k}_{N i}^{T} \dot{\boldsymbol{x}}_{+} & \geq 0 \\
\Delta f_{N i}^{*} \boldsymbol{k}_{N i}^{T} \dot{\boldsymbol{x}}_{+} & =0 \\
\mu \Delta f_{N i}^{*} & \geq\left|\Delta f_{F i}^{*}\right| \\
\Delta f_{F i}^{*} \boldsymbol{k}_{F i}^{T} \dot{\boldsymbol{x}}_{+} & \leq 0 \\
\left(\mu \Delta f_{N i}^{*}-\left|\Delta f_{F i}^{*}\right|\right) \boldsymbol{k}_{F i}^{T} \dot{\boldsymbol{x}}_{+} & =0
\end{aligned}
$$

Here, $\Delta f^{*}$ denotes the optimal solution. $\Delta f_{N i}^{*}$ and $\Delta f_{F i}^{*}$ denote $i$ th solutions of vertical force and friction force, respectively.

The conditions (17)-(19) are related to vertical force. (17) corresponds to a non-negative condition of vertical force, and (18) is equal to a non-negative condition of vertical velocity at the contact point $\left(\boldsymbol{k}_{N i}^{T} \dot{\boldsymbol{x}}_{+}\right.$ expresses the vertical velocity due to the principle of virtual work). (19) means that the vertical velocity at the contact point becomes zero if the vertical force is not zero.

The conditions (20)-(22) are related to friction force. (20) corresponds to a friction condition, and (21) shows 
that the friction force turns to opposite direction to the horizontal velocity at the contact point. (22) means that the friction condition becomes active $\left(\left|\Delta f_{F i}^{*}\right|=\right.$ $\left.\mu \Delta f_{N i}^{*}\right)$ if the contact point slips. If the friction condition is not active $\left(\left|\Delta f_{F i}^{*}\right|<\mu \Delta f_{N i}^{*}\right)$, the horizontal velocity at the contact point becomes zero.

From these conditions, it is confirmed that the plastic collision force realizes the ideal constrained motion.

\section{Control System for Autonomous Walking}

In order to control a non-linear system such as a biped walking robot, we need to know its dynamical behavior and to calculate the interference torque among the joints. In the field of robot manipulator control, the on-line control scheme called computed torque method has been proposed ${ }^{[13]}$, in which the interference torque among other joints can be estimated using the inverse dynamics calculation. It takes, however, much calculation cost and is not suitable for on-line control.

On the other hand, robust-servo based manipulator control scheme has been proposed ${ }^{[6]}$, which can compensate the interference torque as a local disturbance using disturbance observer. As the results, only the kinematics is taken into account in the motion controller and it takes the less calculation cost than computed torque method. Moreover, it has good performance against payload changes.

In this paper, such a robust-servo control is applied to each joint of a biped walking robot. The joint-space reference is transformed from work-space reference using resolved motion rate method ${ }^{[5]}$. Then, the global system is autonomously stabilized by work-space reference generator. The proposed control method has the hierarchical structure.

\subsection{Bottom Level : Robust-servo}

We have proposed a robust-servo control method based on a combination of two-degree-of-freedom control and sliding mode control ${ }^{[16]}$, which has an advantage in the robustness against a disturbance, compared with conventional sliding mode control ${ }^{[7]}$ or disturbance observer based robust control ${ }^{[6]}$.

The configuration of this servo-system is shown in Fig.3. Here, $u, r, y$, and $d$ are the controller's output, the command input, the plant's output, and the disturbance, respectively. $P(s)$ and $P_{n}(s)$ denote the plant and its nominal system. $S(s)$ denotes free-parameter which corresponds to the sensitivity function of the disturbance.

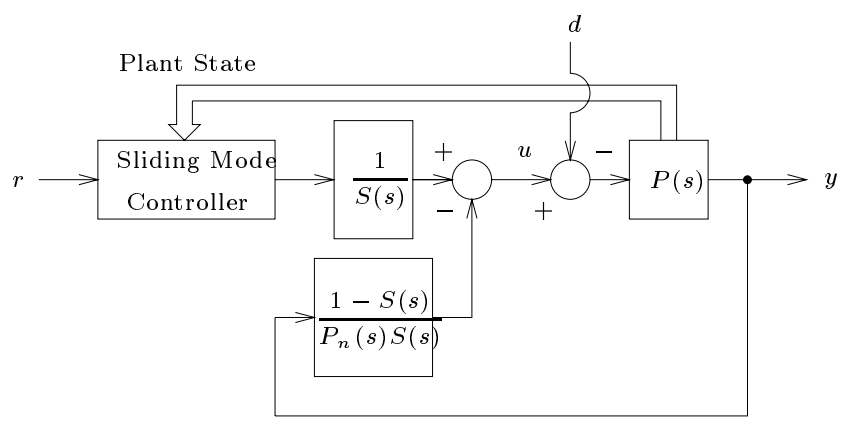

Figure 3: Configuration of 2DOF control system with sliding mode.

In a case of DC servo motor control, the plant system can be regarded as a following 2 nd order system.

$$
y=\frac{K}{(J s+B) s}(u-d)
$$

The global system controlled by the proposed method follows the next 1 st order dynamics ${ }^{[16]}$.

$$
y=\frac{c}{s+c} r-\frac{K_{n}}{J_{n}(s+c)} T_{s} S(s) d^{\prime}
$$

Here, $T_{s}$ denotes the switching time of the VSS, and $d^{\prime}$ is defined as the total disturbance including fluctuations of plant parameters. When the free-parameter $S(s)$ is set to a low gain in the low-frequency domain and the switching time $T_{s}$ is taken enough short, the system (24) becomes very insensitive against the disturbance and the command input response precisely corresponds to the designed trajectory.

Moreover, this sliding controlled system can be reduced the order of the system, that is, 2nd order motion of joint becomes 1st order one, thus it is suitable for an inverse kinematics transformation using a resolved motion rate ${ }^{[5]}$. It takes the less calculation cost than the resolved-acceleration method ${ }^{[4]}$.

\subsection{Middle Level : Inverse Kinematics}

When the robust-servo systems are applied to each joint of biped robot and the position references are set to the current states, it becomes velocity controller. Thus its joint references can be transformed from workspace references by using the resolved motion rate. In this paper, the resolved motion rate method ${ }^{[5]}$ is generalized to control both of the rate of the center of gravity and the rate of the tip of non-support leg at the same time.

When the center of gravity $\boldsymbol{x}_{c}$ and the tip position of non-support leg $\boldsymbol{x}_{t}$ can be expressed as following 
function of joint angle $\boldsymbol{q}$ with respect to the origin of the support foot,

$$
\begin{aligned}
& \boldsymbol{x}=\boldsymbol{f}(\boldsymbol{q}) \\
& \text { where } \quad \boldsymbol{x}^{T}=\left[\begin{array}{lll}
\boldsymbol{x}_{c}^{T} & \boldsymbol{x}_{t}^{T} & x_{g}
\end{array}\right] \\
& \boldsymbol{x}_{c}: \text { center of gravity } \\
& x_{t} \quad \text { : tip position of non-support leg } \\
& x_{g} \quad \text { : gradient of body }
\end{aligned}
$$

the derivatives become,

$$
\dot{\boldsymbol{x}}=J_{a c o} \dot{\boldsymbol{q}}
$$

Here, $J_{a c o}$ is jacobian matrix about the center of gravity, the tip position of non-support leg, and the gradient of body. This jacobian matrix is algebraically very complex but they can be easily obtained by using a mathematics software package ${ }^{[17]}$.

Considering (27) as restrictions, we can calculate the joint space references $\dot{\boldsymbol{q}}^{\text {ref }}$ from work space references $\dot{\boldsymbol{x}}^{\text {ref }}$ by solving a following QP problem.

$$
\begin{aligned}
\text { minimize } & \frac{1}{2} \dot{\boldsymbol{q}}^{\text {ref }{ }^{T}} \boldsymbol{C} \dot{\boldsymbol{q}}^{r e f}-\boldsymbol{c}_{0}^{T} \dot{\boldsymbol{q}}^{\text {ref }} \\
\text { subject to } & J_{a c o} \dot{\boldsymbol{q}}^{r e f}=\dot{\boldsymbol{x}}^{r e f}
\end{aligned}
$$

Where $\boldsymbol{C}$ and $\boldsymbol{c}_{0}$ can be defined at designer's will, but usually $\boldsymbol{C}$ is set to an identity matrix and $\boldsymbol{c}_{0}$ is set to zero.

In order to control the position, the velocity reference $\dot{\boldsymbol{x}}^{\text {ref }}$ is generated by following equation.

$$
\dot{\boldsymbol{x}}^{r e f}=K_{p}\left(\boldsymbol{x}^{c m d}-\boldsymbol{x}\right)+\dot{\boldsymbol{x}}^{c m d}
$$

Here, diagonal matrix $K_{p}$ is proportional gain.

All of mechanical characteristics are expressed only in this transformation.

\subsection{Top Level : Motion Design for Au- tonomous Stamping}

The most important issue in the biped walking control is the motion design, that is, the trajectory design of the center of gravity and the tip position of non-support leg. Those trajectories should be autonomously generated from the sensor informations.

The motion of center of gravity should essentially obey the dynamics of inverted pendulum because the biped robot has its mode. If the reference command is generated ignoring the dynamics of inverted pendulum, it causes the flotage of the support foot and then the system becomes uncontrollable. Some control methods have been proposed from this viewpoint ${ }^{[10][11]}$.
The reference of the center of gravity should satisfy the following dynamics of an inverted pendulum mode with linear approximation.

$$
\begin{aligned}
& \ddot{x}_{c 1}=\omega^{2} x_{c 1}+u_{v 1} \\
& \ddot{x}_{c 2}=\omega^{2} x_{c 2}+u_{v 2}
\end{aligned}
$$

Here, $x_{c 1}$ and $x_{c 2}$ denote the center of gravity in sagittal plane and lateral plane with respect to the origin of the support foot, respectively. $u_{v}$ denotes a virtual input which has a limit to prevent flotage of the foot. $\omega$ corresponds to $\sqrt{g / l}, l$ is the length of the pendulum, and $g$ is the gravity constant.

First, we consider the stabilization in the lateral plane, that is, the stable stamping with an arbitrary frequency. The frequency control of center of gravity in the lateral plane is very important because another principal references can be generated using the states of its vibration. In order to control the stamping frequency, we introduce a kind of energy of the system (32) as follows,

$$
E_{2}=\frac{1}{2} \dot{x}_{c 2}^{2}-\frac{1}{2} \omega^{2} x_{c 2}^{2}
$$

If the above energy is constant and there are no losses at the time of leg support change, the stamping frequency has some constant value. Let $x_{c 2}(0)$ be the offset of center of gravity with respect to the origin of the support foot at the time of leg support change (see Fig.4), the solution of (32) without input $u_{v 2}$ becomes,

$$
\begin{aligned}
x_{c 2}(t)= & \frac{1}{2}\left\{\left(x_{c 2}(0)+\frac{\dot{x}_{c 2}(0)}{\omega}\right) e^{\omega t}\right. \\
& \left.+\left(x_{c 2}(0)-\frac{\dot{x}_{c 2}(0)}{\omega}\right) e^{-\omega t}\right\}
\end{aligned}
$$

And then, the stamping period $T$ is defined as following equation.

$$
\begin{aligned}
& x_{c 2}(T / 2)=x_{c 2}(0)=W_{2} / 2 \\
& \dot{x}_{c 2}(T / 2)=-\dot{x}_{c 2}(0)
\end{aligned}
$$

Here, $W_{2}$ denotes the width of both legs (constant).

From (33)-(36), we can obtain the relationship between the energy $E_{2}$ and the stamping period $T$ as follows,

$$
E_{2}=-\frac{\omega^{2} W_{2}^{2} e^{\frac{\omega T}{2}}}{2\left(1+e^{\frac{\omega T}{2}}\right)^{2}}
$$

Therefore, we may control the energy $E_{2}$ in place for controlling the stamping period $T$.

Next, we describe how to determine the virtual input $u_{v 2}$ to control the energy $E_{2}$. The derivative of the energy $E_{2}$ can be calculated as follows,

$$
\dot{E}_{2}=\ddot{x}_{c 2} \dot{x}_{c 2}-\dot{x}_{c 2} \omega^{2} x_{c 2}=\dot{x}_{c 2} u_{v 2}
$$




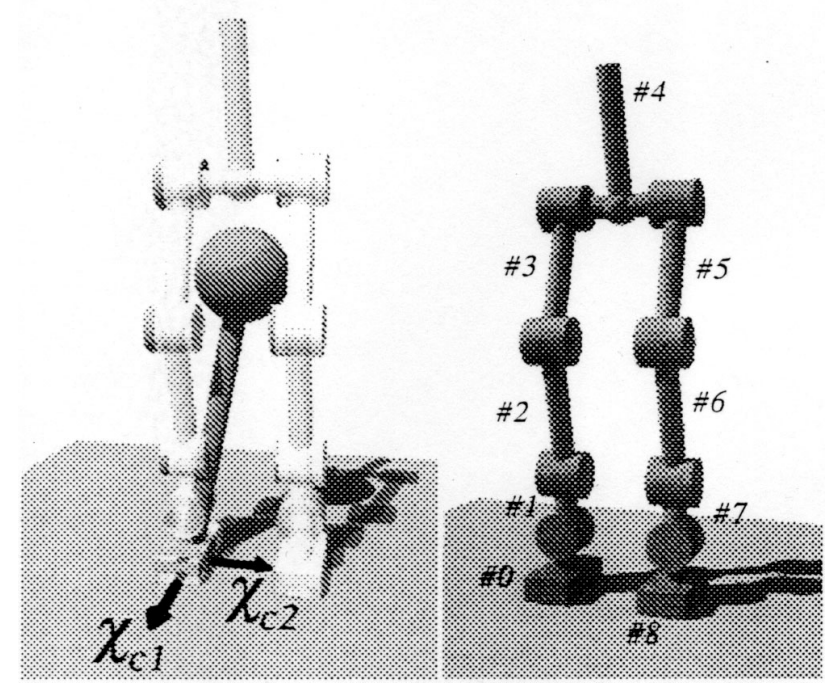

Figure 4: Simplified model of inverted pendulum and 8 -axis biped robot.

The derivative of the energy $E_{2}$ and the virtual input $u_{v 2}$ has a kind of the linear relationship. Then we determine the virtual input $u_{v 2}$ as follows,

$$
\begin{aligned}
u_{v 2} & =\frac{k_{E}}{\dot{x}_{c 2}}\left(E_{2}^{r e f}-E_{2}\right) \\
E_{2}^{r e f} & =-\frac{\omega^{2} W_{2}^{2} e^{\frac{\omega T^{r e f}}{2}}}{2\left(1+e^{\frac{\omega T^{r e f}}{2}}\right)^{2}}
\end{aligned}
$$

Under this control, the dynamics of energy $E_{2}$ becomes,

$$
\dot{E}_{2}=-k_{E} E_{2}+k_{E} E_{2}^{r e f}
$$

The energy $E_{2}$ can become equal to its reference $E_{2}^{r e f}$ if the value of $k_{E}$ is chosen so that the characteristic roots of (41) has negative real part.

Then, the walking velocity in sagittal plane can be also controlled by similar method. Using the same energy control method, the arbitrary step width $W_{1}^{\text {ref }}$ can be realized by following equation.

$$
E_{1}^{r e f}=\frac{\omega^{2} W_{1}^{r e f^{2}} e^{\frac{\omega T^{r e f}}{2}}}{2\left(1-e^{\frac{\omega T^{r e f}}{2}}\right)^{2}}
$$

Then, the reference of tip position of non-support leg can be generated from the motion of gravity center. The height position reference of non-support leg $x_{t 3}^{r e f}$ can be generated as the following function of gravity center in lateral plane.

$$
x_{t 3}^{r e f}=f_{h}\left(\left|x_{c 2}\right|-W_{2} / 2\right)
$$

Here, the function $f_{h}(\cdot)$ is shown in Fig.5. The position in sagittal plane can be also generated as the function of the gravity center in sagittal plane.

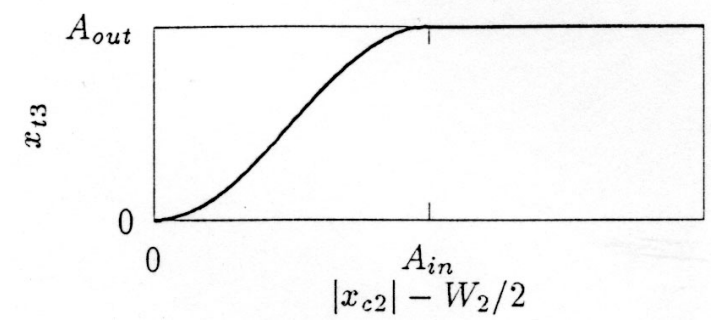

Figure 5: Nonlinear projection from gravity center to foot height reference.

\section{Simulation Results}

A simulation result of stabilization of a walking 8axis biped robot is shown in Fig.6. The configurations of the biped model are also shown in Fig. 4 and Table 1. In this simulation, the stamping time and the walking velocity is set to $0.6[\mathrm{sec}]$ and $0.2[\mathrm{~m} / \mathrm{s}]$. The trajectory of gravity center is shown in Fig.7 and about $2[\mathrm{~m}]$ walking can be observed. In this case, it is difficult to stabilize the motion in sagittal plane because the motion of non-support leg makes bad influence on the inverted pendulum mode of gravity center. In the future work, we need to modify the approximation (31) to improve the walking motion in sagittal plane. The position of center of gravity in the lateral plane is shown in Fig.8, and Fig.9 shows its phase plane trajectory. This stamping motion has a stable limit cycle in the lateral plane.

\begin{tabular}{lcc}
\hline link & size $(d \times w \times h)[\mathrm{m}]$ & weight $[\mathrm{kg}]$ \\
\hline$\#$ 0, \# 8 & $0.2 \times 0.1 \times 0.05$ & 1.0 \\
$\# 1, \#$ 7 & $0.1 \times 0.1 \times 0.05$ & 0.5 \\
$\#$ 2, \# 3, \#5, \# 6 & $0.1 \times 0.1 \times 0.2$ & 2.0 \\
$\#$ 4 & $0.1 \times 0.1 \times 0.3$ & 3.0 \\
\hline total & $0.3 \times 0.2 \times 0.8$ & 14.0 \\
\hline
\end{tabular}

Table 1: Size of the robot.

\section{Conclusion}

First, the three dimensional precise digital simulation method is proposed, which is the extension of open link manipulator simulation method and contact simulation method of rigid body mechanics. This simulation model can be said to be mathematically exact, thus the essential investigation of biped control algorithms is available. This approach takes the less cost than the experimental demonstration. 

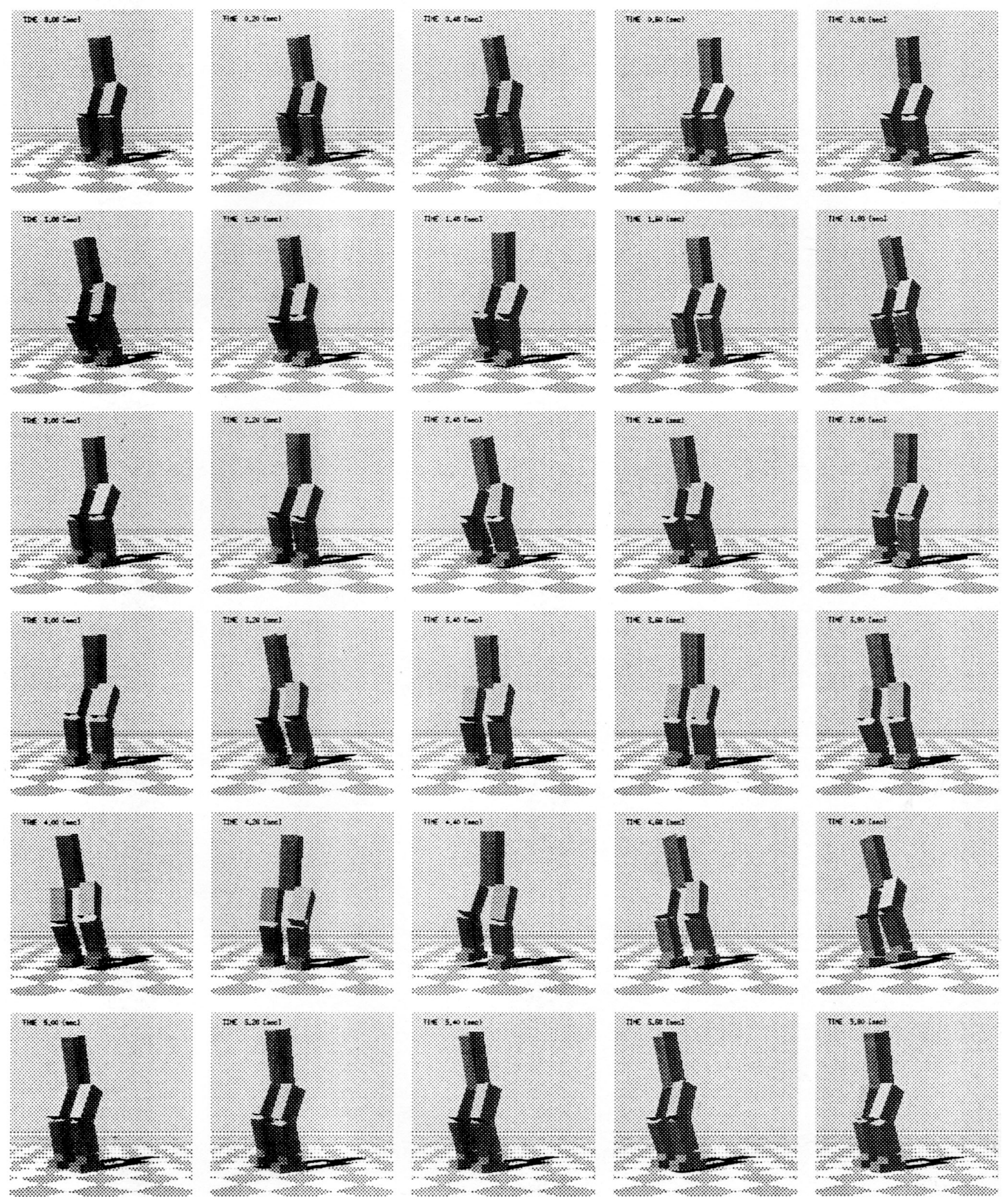

Figure 6: Pictures of stamping biped robot. 


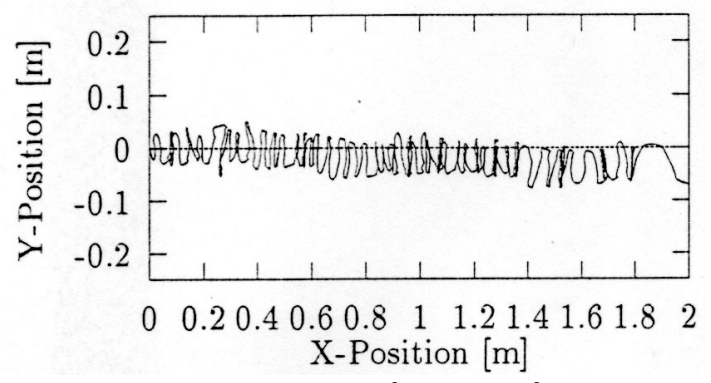

Figure 7: Trajectory of center of gravity

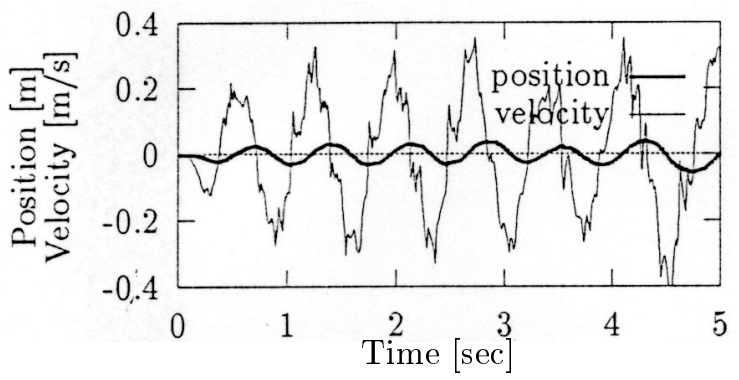

Figure 8: Position and velocity in lateral plane.

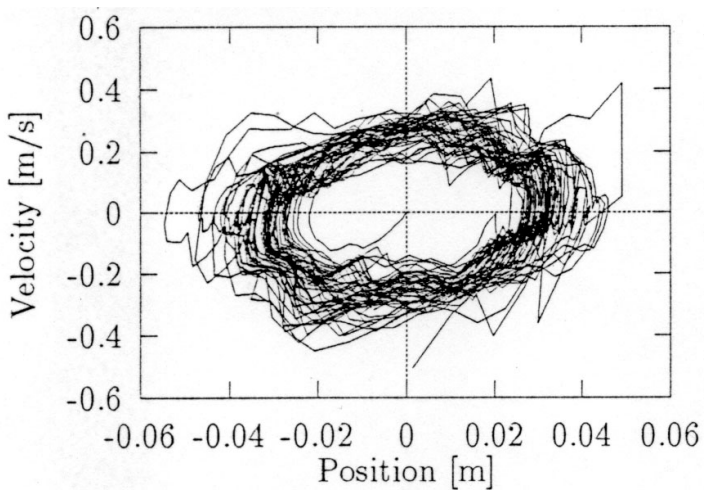

Figure 9: Phase portrait in lateral plane.

Second, the autonomous walking control with hierarchical structure has been proposed and inspected by the simulation. The references of arbitrary frequency walking motion are autonomously generated from the vibration motion of the center of gravity in lateral plane. Both of references, the center of gravity and the tip position of non-support leg, can be simultaneously transformed to the joint space reference, and the robust-servo controllers are applied.

Third, the existence of the limit cycle of the dynamical stable stamping motion has been confirmed in the simulations.

Authors believe that the proposed autonomous walking control scheme and the proposed simulation method will open a new field of biped robot researches.

\section{References}

[1] H. Hemami and R. L. Farnsworth, "Postural and Gait Stability of a Planar Five Link Biped by Simulation", IEEE Trans. Automatic Control, Vol. 22, no. 3, pp. 452-458, 1977.

[2] H. Hemami and B. F. Wyman, "Modelling and Control of Constrained Dynamic System with Application to Biped Locomotion in Frontal Plane", IEEE Trans. Automatic Control, Vol. 24, no. 4, pp. 526-535, 1979.

[3] L. Shih, A. A. Frank and B. Ravani, "Dynamic Simulation of Legged Machines Using a Compliant Joint Model", Int. J. Robotics Research, Vol. 6, no. 4, pp. 33-46, 1987.

[4] J. Y. S. Luh, M. W. Walker and R. P. C. Paul, "ResolvedAcceleration Control of Mechanical Manipulators", IEEE Trans. Automatic Control, Vol. 25, no. 3, pp. 468-474, 1980.

[5] D. E. Whitney, "Resolved Motion Rate Control of Mechanical Manipulators and Human Prostheses", IEEE Trans. Man-Machine Systems, Vol. 10, no. 2, pp. 47-53, 1969.

[6] K. Ohnishi and T. Murakami, "Advanced Motion Control in Robotics", Proc. IEEE IECON'89, pp. 356-359, 1989.

[7] V. I. Utkin, "Variable Structure Systems with Sliding Modes", IEEE Trans. Automatic Control, Vol. 22, no. 2, pp. 212-222, 1977.

[8] J. Yamaguchi, A. Takanishi and I. Kato, "Development of a Biped Walking Robot Compensating for Three-Axis Moment by Trunk Motion" J. Robotics Society of Japan, Vol. 11, no. 4, pp.581-586, 1993. (in Japanese)

[9] S. Kawaji, N. Matsunaga and M. Arao, "Hierarchical Control of Biped Locomotion Robot", Proc. IEEE Int. Workshop on Advanced Motion Control'94, pp. 421-430, 1994.

[10] J. Furusho and A. Sano, "Sensor-Based Control of a NineLink Biped", Int. J. Robotics Research, Vol. 9, no. 2, pp. 83-98, 1990.

[11] H. Minakata and Y. Hori, "Realization of Robust Biped Walking by Emulating Inverted Pendulum", Proc. IEEE Int. Workshop on Advanced Motion Control'94, pp. 460467, 1994.

[12] M. W. Walker and D. E. Orin, "Efficient Dynamic Computer Simulation of Robotic Mechanisms", ASME J. Dynamic Systems, Measurement, Control, Vol. 104, pp. 205211, 1982 .

[13] J. Y. S. Luh, M. W. Walker and R. P. C. Paul, "Online Computational Scheme for Mechanical Manipulators", ASME J. Dynamic Systems, Measurement, Control, Vol. 102, pp. 69-76, 1980.

[14] P. Lötstedt, "Numerical Simulation of Time-Dependent Contact and Friction Problems in Rigid Body Mechanics", SIAM J. Scientific and Statistical Computing, Vol. 5, no. 2, pp. 370-393, 1984.

[15] R. W. Cottle and G. B. Dantzig, "Complementary Pivot Theory of Mathematical Programming", Linear Algebra and Its Applications, Vol. 1, pp. 103-125, 1968.

[16] Y. Fujimoto and A. Kawamura, "Robust Servo-system Based on Two-Degree-of-Freedom Control with Sliding Mode", Proc. IEEE IECON'93, pp. 85-90, 1993.

[17] for example, S. Wolfram, MathematicaTM - A System for Doing Mathematics by Computer, Addison-Wesley Publishing, 1992 . 